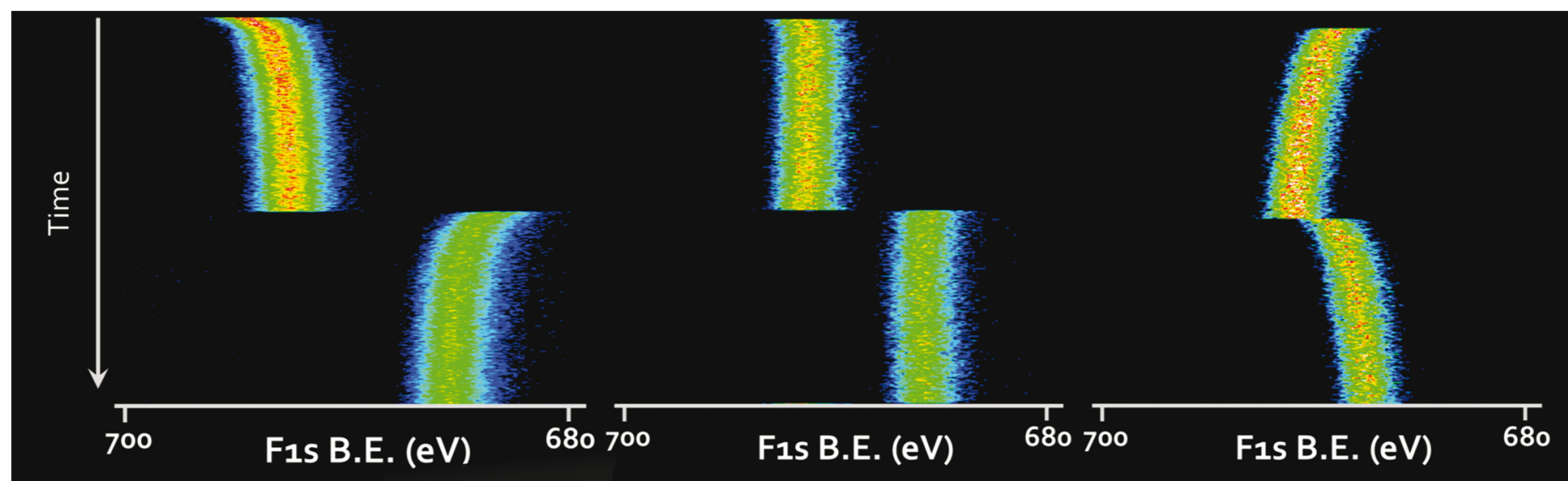

X-ray

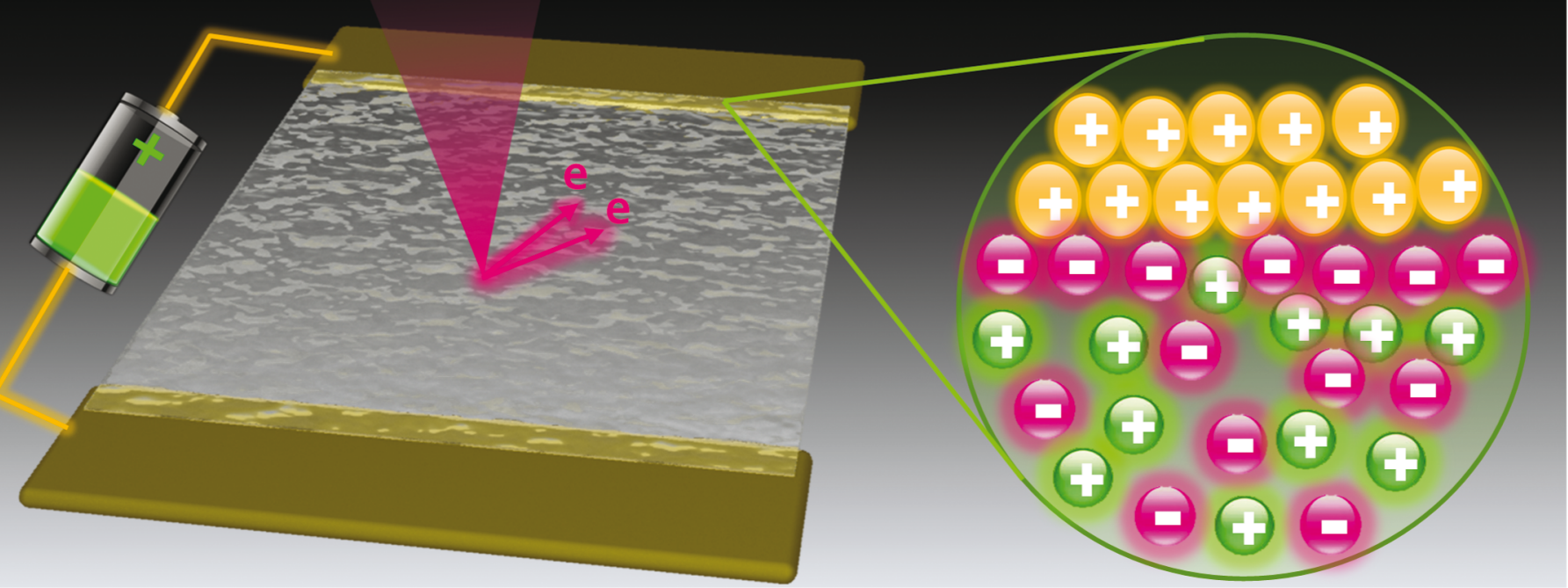

Showcasing research from the surface-science laboratory of Dr Sefik Suzer at the Chemistry Department of Bilkent University, Ankara, Turkey.

Title: XPS enables visualization of electrode potential screening in an ionic liquid medium with temporal- and lateral-resolution

X-ray photoelectron spectroscopy is used to map local electrical potentials in a device configured as a coplanar capacitor, with an ionic liquid infused porous polymer film between two electrodes. The screening of the electrodes in response to voltage bias is followed by time- and position-dependent F1s signals of the ionic liquid millimeters away from the electrodes. Shifts in the binding energy of the peaks reveal that the device has two oppositely polarized regions, each following the polarization of one of the electrodes.

\section{As featured in:}

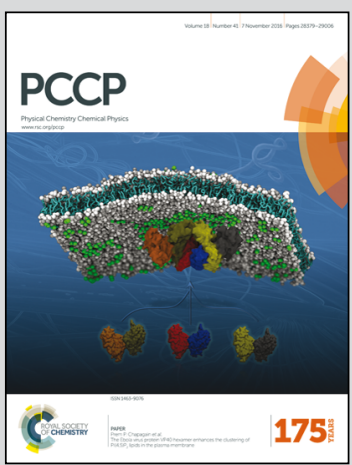

See S. Suzer et al.,

Phys. Chem. Chem. Phys., 2016, 18, 28434. 
CrossMark \& click for updates

Cite this: Phys. Chem. Chem. Phys., 2016, 18, 28434

Received 15th July 2016, Accepted 15th August 2016

DOI: 10.1039/c6cp04933h

www.rsc.org/pccp

\title{
XPS enables visualization of electrode potential screening in an ionic liquid medium with temporal- and lateral-resolution $\dagger$
}

\author{
M. T. Camci, ${ }^{a}$ P. Aydogan, ${ }^{a}$ B. Ulgut, ${ }^{a}$ C. Kocabas ${ }^{b}$ and S. Suzer ${ }^{\star a}$
}

We present an X-ray photoelectron spectroscopic (XPS) investigation of potential screening across two gold electrodes fabricated on a porous polymer surface which is impregnated with the ionic liquid (IL) $\mathrm{N}-\mathrm{N}$-diethyl-N-methyl- $\mathrm{N}$-(2-methoxyethyl)ammonium bis(trifluoromethanesulfonyl)imide [DEME-TFSI]. The IL provides a sheet of conducting layers to the insulating polymer film, and allows monitoring charging and screening dynamics at the polymer + IL/vacuum interface in a laterally resolved fashion across the electrodes. Time-resolved measurements are also implemented by recording F1s peaks of the $\mathrm{IL}$, while imposing $10 \mathrm{mHz}$ square-wave (SQW) pulses across the two electrodes in a sourcedrain geometry. Variations in the F1s binding energy reflect directly the transient local electrical potential, and allow us to visualize screening of the otherwise built-in local voltage drop on and across the metal electrodes in the range of millimeters. Accordingly, the device is partitioned into two oppositely polarized regions, each following polarization of one electrode through the IL medium. On the other extreme, upon imposing relatively fast $1 \mathrm{kHz}$ SQW pulses the charge screening is prevented and the device is brought to assume a simple resistor role. A simple equivalent circuit model also reproduces the observed voltage transients qualitatively. The presented structure and variants of XPS measurements, enabling us to record voltage transients in unexpectedly large lateral distances away from the electrodes, can impact the understanding of various electrochemical concepts.

\section{Introduction}

A colossal research activity has been devoted to ionic liquids (ILs) since they offer unique physical and chemical properties towards numerous advanced applications. ${ }^{1,2}$ In particular, the use of ILs for electrochemical applications has been in focus for energy storage, such as super-capacitors and/or battery materials, ${ }^{3-5}$ in single molecule detection, ${ }^{6}$ and colorimetric sensing. ${ }^{7}$ In all cases, furthering the understanding of charge accumulation and dissipation on electrode-electrolyte interfaces is of utmost importance. It is well-known that when an electrified solid interface is brought into contact with ionic conductors, the surface accumulates opposite charges and forms an electrical double layer (EDL) at the interface, and numerous advanced electrochemical, spectroscopic, microscopic and theoretical/ simulation techniques have been employed towards advancing the basic understanding of the interface properties of ILs,

\footnotetext{
${ }^{a}$ Department of Chemistry, Bilkent University, Ankara 06800, Turkey.

E-mail: suzer@fen.bilkent.edu.tr; Tel: +90-312-290 1476

${ }^{b}$ Department of Physics, Bilkent University, Ankara 06800, Turkey

$\dagger$ Electronic supplementary information (ESI) available: Additional XP spectra and current measurements. See DOI: 10.1039/c6cp04933h
}

especially when they are electrified. ${ }^{8-34}$ As a result of these advanced research efforts, the structure of the double layer down to nanometer scales, and especially intricate changes at interfaces accompanying their electrification, has now been brought up to a new level of understanding, and this subject has recently been reviewed. ${ }^{35}$ Accordingly, at the steady-state the double layer in ILs consists of pronounced charge density oscillations within a short range (1-10 nm) from the electrode surface, which can also be controlled by the sign and/or magnitude of the electrification. By recording electrostatic surface forces, it was recently reported that electrostatic screening can even have a longer range component up to $10-40 \mathrm{~nm} .^{36}$ Another interesting finding was reported by Liu et al., ${ }^{37}$ where asymmetric ion distribution was found to extend to micron ranges under electrostatic-actuation in ionic polymer actuators containing ILs.

Dynamic information related to changes in the interface structure and properties when subjected to electrical pulses has also been investigated using transient electrochemical current and capacitance as well as transient interfacial tension measurements using electrocapillarity. ${ }^{38,39}$ These findings were also supported by various spectroscopic studies, which revealed an extremely slow ionic rearrangement and an accompanying hysteresis. ${ }^{40,41}$ 
In the present contribution we will show that transient effect(s) of screening of the electrode potentials by ILs can also be measured in similar time domains up to minutes, but at the same time, we will also show that the voltage transients can be measured in a completely surprising and different realm, i.e. up to millimeter ranges away from the interface, using X-ray photoelectron spectroscopy (XPS).

Monitoring charge accumulation/depletion is an outstanding challenge, and XPS, although an ultra-high vacuum analysis technique, provides chemically specific information for also ILs, due to their low vapor pressure together with moderate ionic conductivity at room temperature. Therefore, XPS has been extensively utilized for probing various physicochemical properties of ILs as well as their interfaces, over the last decade. ${ }^{42-51}$ In addition to chemical specificity, XPS can also provide information related to certain electrical properties of surface structures, which has been exploited by us and others. ${ }^{52-54}$ This is achieved by the application of some form of an electrical stimulus to or across the sample in order to polarize the surfaces. Imposing a potentiostatically controlled DC bias using an electrochemical set-up and recording XPS were first shown for ILs by FoelskeSchmitz et $a l^{55-57}$ In the present manuscript, we present a similar XPS investigation, but extend it further for probing dynamics of potential screening both in a position- as well as time-dependent fashion across two metal electrodes of a device which has an ionic liquid medium in between. One of the metal electrodes is subjected to various DC and/or AC electrical pulses to induce static and dynamic polarization, respectively, while the other electrode is grounded. In parallel with these electrical interventions, XPS data are recorded either in the snap-shot mode with $1 \mathrm{~s}$ time intervals to probe transients, and lower energy resolution, or in conventional longer scanning mode with higher resolution for probing the steady-state behavior, depending on the information sought-after. Data are also recorded in the line-scan mode to obtain position dependent information across the electrodes.

\section{Experimental}

The device was fabricated by depositing two gold electrode patches with the same geometry on a $20 \mu \mathrm{m}$ thick porous polyethylene membrane (PEM) (Celgard 2730, Gelon LIB Group) with a porosity of $43 \%$. Afterwards, the membrane with electrodes is fixed onto a glass substrate for electrical isolation from the sample holder. Electrochemistry grade ionic liquid $(\geq 98.5 \%)$ DEME-TFSI $[N, N$-diethyl- $N$-methyl- $N$-(2-methoxyethyl)ammonium bis(trifluoromethanesulfonyl)imide] was purchased from SigmaAldrich (CAS number: 464927-84-2). $5 \mu \mathrm{l}$ of DEME-TFSI was injected to the surface of the PEM between the Au-electrodes, which spreads rapidly and wets even the electrodes. To minimize moisture absorption by the IL, the device, together with ILs, is quickly inserted to the load-lock of the instrument. Pumping is started immediately and continued for more than 12 hours. Other ILs have also been used in a similar device geometry, but only the results of DEME-TFSI will be presented in this work.
A Thermo Fisher K-Alpha X-ray Photoelectron Spectrometer with a monochromatized photon energy of $1486.6 \mathrm{eV}$ is used for all XPS measurements, for samples of 5-10 nm depth. However, the lateral resolution is limited to the X-ray spot size which can be focused down only to $30 \mu \mathrm{m}$. Even under focused beam, we have not detected significant X-ray damage, also in prolonged measurement times of up to 2 days. The device is connected, through the metal electrodes, either to ground or to a DC source for static XPS analysis or to a DS340 Function Generator (Stanford Research) for imposing AC electrical stimuli, and harvesting dynamical information. Spectra have been recorded using various amplitudes and frequencies, but only 3 and $5 \mathrm{~V}$ results will be shown here, which were chosen for spectral clarity. In addition to conventional scanning mode, the instrument can also be used in the snap-shot mode with a moderate time resolution down to 0.2 to $1 \mathrm{~s}$, depending on the signal strength.

\section{Results and discussion}

The device and the experimental set-up are depicted in Fig. 1, together with a survey XP spectrum of the IL medium, which also confirms the integrity and the stoichiometry of the IL. This point is elaborated further in Fig. S1 and S2 in the ESI. $\dagger$ Assuming the probed surface composition to be uniform, we can estimate the probed top (5-10 nm) of the surface to contain one ion-pair of the IL for every $\sim 60$ carbon atoms of the polymer membrane, which means that the final IL medium within the device is also thin. This is obtained by using the relative intensity ratio of the two strong peaks observed, i.e. F1s/ C1s, and working backwards.

Imposing a voltage stimulus induces an exponentially decaying current with time constants in tens of seconds as given in Fig. S3 in the ESI. $\dagger$ At prolonged times $(>1000 \mathrm{~s})$ this current reaches a steady-state value of $\sim 10 \mathrm{nA}$ (not shown), which could be thought of as a leaky capacitor where the main leakage mechanism is expected to be oxidation and reduction taking place on the gold surface of the electrodes. For example, we observed that the prolonged application of $+3 \mathrm{~V}$ bias leads to the formation of Au-nanoparticles in the vicinity of the polarized electrode but within the ionic liquid medium, which will be the subject of our next publication.

The imposed potential is screened in time through the formation of the double layer by the mobile ions of the IL medium, which can be followed by XPS measurements. Note that, even though our lateral resolution $(30 \mu \mathrm{m})$ is not capable of probing the EDL directly, its influence is easily probed by measuring the binding energy position of the F1s, or any other peak of the IL, as shown in Fig. 2. As revealed by the figure, a steady-state surface potential is established after the formation of the EDL, which is uniform, in the millimeter range, throughout the entire surface of the IL medium. We have analyzed more than 10 similar devices, with slightly different F1s intensity distribution, but always exhibiting a uniformly shifted constant binding energy position. This is the first surprising 


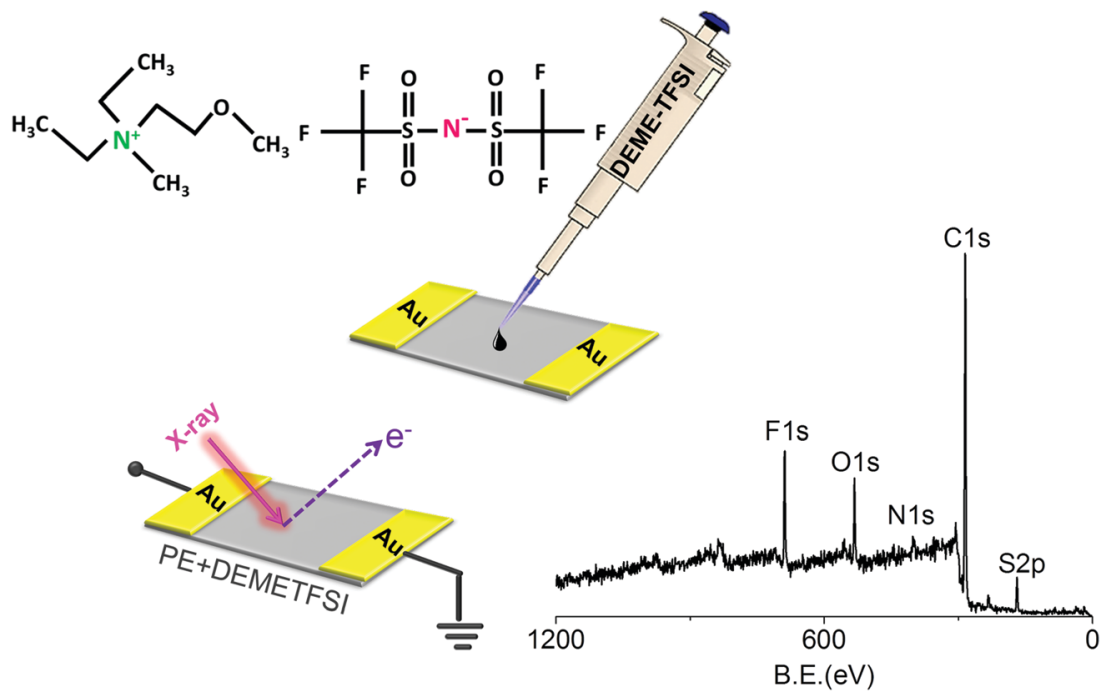

Fig. 1 Experimental set-up and a survey XP spectrum of the IL and polymer membrane.
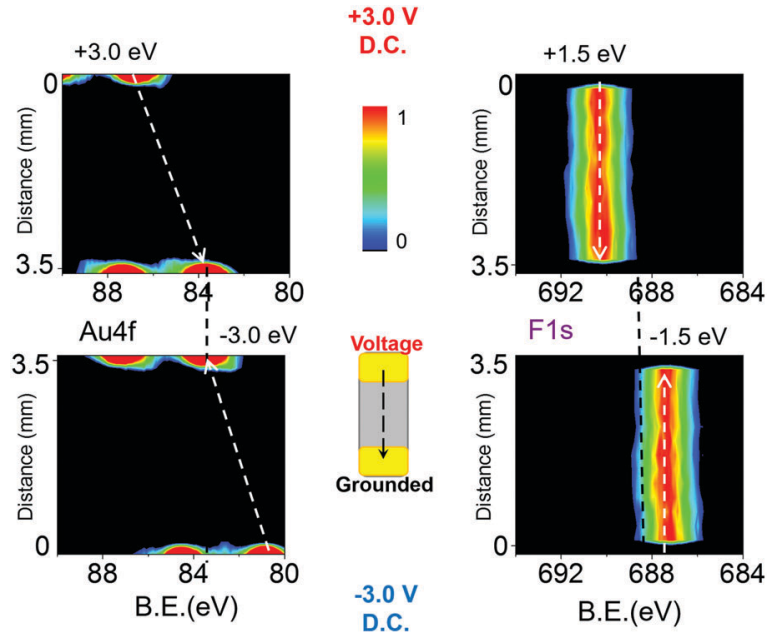

Fig. 2 XP spectra of Au4f and F1s regions, recorded in the line scan mode between the two gold electrodes, containing a porous polymer film impregnated with the ionic liquid DEME-TFSI, under the application of +3 (upper part) or $-3 \vee$ (lower part) DC bias. The intensity of the F1s peak shows some fluctuations along the line, and therefore the spectra shown are normalized.

finding, since a similar device containing a graphene layer in between the electrodes behaves totally different as was reported by us. ${ }^{53}$ In that work, we measured the C1s peak position and it was found to exhibit a smoothly and linearly varying (not constant) value across the two electrodes, acting like a simple resistive strip. Similar data are reproduced in Fig. S4 in the ESI. $\dagger$

When grounded the binding energy of the $A u 4 f_{7 / 2}$ peak is $84.0 \mathrm{eV}$. Under DC bias, and after equilibrium is established, the corresponding values are measured, at the biased electrode, to be 87.0 and $81.0 \mathrm{eV}$ when $+3 \mathrm{~V}$ and $-3 \mathrm{~V}$ are applied to it, respectively, complying totally the applied bias (i.e. exactly $3.0 \mathrm{eV}$ shift). Naturally, no corresponding shift is observed at the grounded electrode. However, as mentioned above, the F1s and also the other IL peaks such as N1s (given in Fig. S5 in the ESI $\dagger$ )

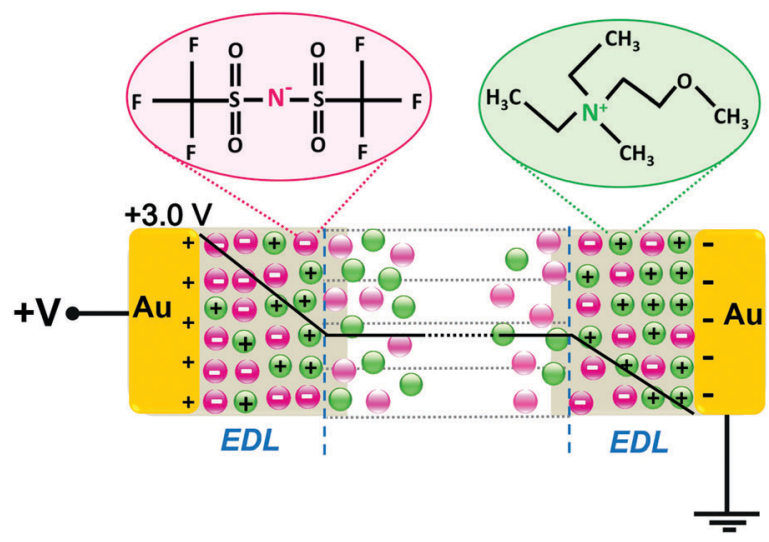

Scheme 1 Schematic representation of the device under $+3 \vee D C$ bias, which is screened at both interfaces by the two EDLs formed.

exhibit a constant, flat, and non-varying shift across the electrodes. Moreover, the measured shift is only $1.5 \pm 0.1 \mathrm{eV}$, and corresponds to only half of the imposed potential, i.e. from the grounded reference value of the $688.5^{47}$ to 690.0 and $687.0 \mathrm{eV}$, under $+3 \mathrm{~V}$ and $-3 \mathrm{~V}$ bias, respectively. Thus, only half of the imposed potential prevails on the IL surface, and the rest is partitioned equally to the two metal electrode-IL interfaces, as depicted in Scheme 1. Note that this symmetry is justified due to the similar nature and the geometry of the gold electrodes. This important finding differs also from that reported by Foelske-Schmitz et al. ${ }^{55-57}$ where a potentiostatic arrangement was used and the potential measured was reported against the quasi-reference electrode of a three electrode cell. In their arrangement the measurement of the potential was conducted in a circuit loop where no current flows (i.e. between reference electrode and the working electrode), and the current is carried in a different loop going through the active and the counter electrodes. We only have a two electrode cell, and our values refer to the actual potential applied to one of the 
electrodes, and the local potential is measured in a non-contact fashion via the position of the F1s peak. Therefore, the potential we report is the potential across the two electrodes where the current also passes. The points across which the reported potential is measured define the major difference between the two electrode and the three electrode setups. In a three electrode setup, since the potential is measured through a reference electrode where ideally no current flows, the measured voltage only reflects the double layer charging on the working electrode and not the double layer charging on the counter electrode.

Our arrangement is closer to a typical electrolytic coplanar capacitor geometry ${ }^{58,59}$ more accurately than the three electrode geometry that is commonly used to study the theoretical fundamentals. ${ }^{55-57}$ In fact, in an earlier work for simulating interfacial tension and capacitance of an IL in between two coplanar graphite electrodes, a very similar potential distribution was predicted via a simulation by Merlet et al. ${ }^{60}$ Whereas, the main emphasis of their work was on simulation of the voltage induced charge density oscillations down to the subnanometer scale, and observed differential capacitance and surface tension of the fluid, they have also pointed out that only half of the applied potential was to be retained in the bulk IL, between the two electrodes (see Fig. 3 in ref. 60), in total agreement with our observations and also with the model presented by Fujimoto and Awaga. ${ }^{61}$

Applying a time-varying electrical signal in the form of slow square-waves (SQWs) with a frequency of $10 \mathrm{mHz}$ and an amplitude of $5 \mathrm{~V}$ allows to us detect the temporal response of the surface potential by recording the F1s signal (or any other peak) with $1 \mathrm{~s}$ time-resolution, as shown in Fig. 3, and also as a function of the lateral position of the probed region from the electrodes, i.e. with lateral resolution as well. The two electrode

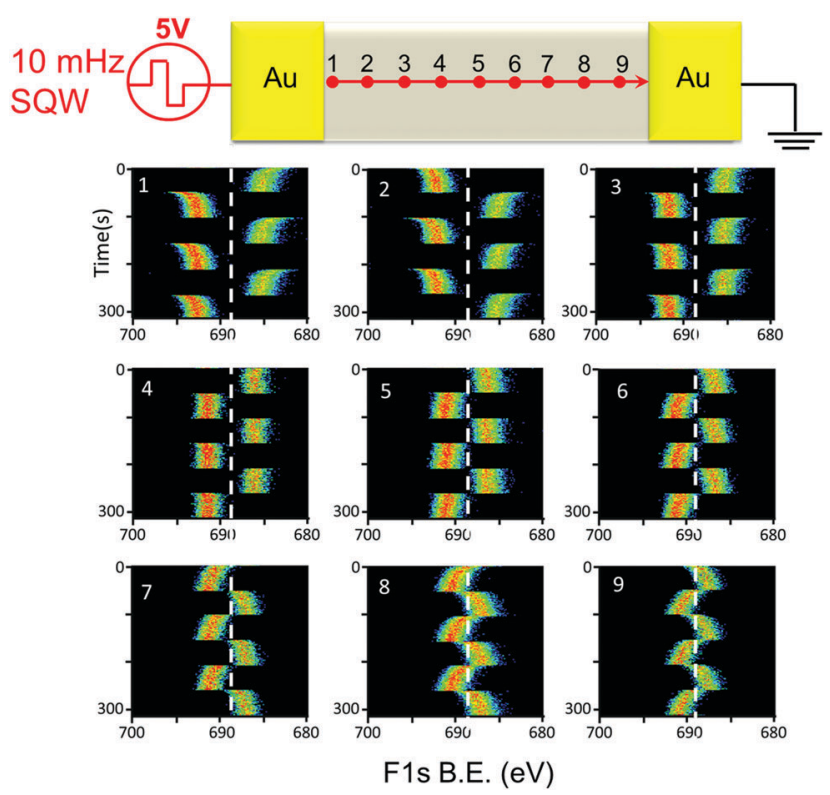

Fig. 3 Time-resolved XP spectra of the F1s region under $10 \mathrm{mHz} 5 \mathrm{~V} \mathrm{SQW}$ excitation and recorded at nine different lateral positions with $\sim 800 \mu \mathrm{m}$ intervals across the gold electrodes, as indicated in the scheme. device, with a conducting IL medium in-between them, provides a platform to study charge screening and dynamics both in a moderate time- and position-resolved fashion. When we apply a voltage bias between the electrodes, the mobile ions of the IL screen the local potential on the electrode and form an EDL. The effects of this screening vary along the channel length, such that it acts in opposite directions at the two electrodes, as depicted in Scheme 1. Moreover, this scheme predicts the presence of a hypothetical point of no motion (in time) near the midpoint of the electrodes, which is also verified experimentally, and is shown in Fig. 3.

In Fig. 4, we reproduced parts of the data presented in the latter figure at positions 1, 4 and 9, respectively, as well as limiting the F1s spectra to the first 1-100 s time-window. When the positive cycle of the $10 \mathrm{mHz}$ SQW excitation is switched on, the F1s peak shifts to $688.5+5.0=693.5 \mathrm{eV}$, upon which the IL starts screening this potential and reduces it down to $691.0 \mathrm{eV}$, the equilibrium potential $(+2.5 \mathrm{~V})$ of the IL medium under $+5 \mathrm{~V}$ electrification. This is exactly what is measured in regions near the electrode as shown in Fig. 4(a). As can also be gathered from the same figure, in the negative cycle (i.e. $50 \mathrm{~s}$ later) the peak starts at $688.5-5.0=683.5 \mathrm{eV}$ and moves towards $686.0 \mathrm{eV}$. However, the regions near the ground electrode behave in the opposite direction, since at the beginning, the interface was negatively polarized, and hence a symmetric but opposite screening is observed as a function of time as shown in Fig. 4(c). The middle point does not experience any screening, and hence no time dependence in the position of the F1s signal is observed [Fig. 4(b)].

By varying the frequency of the SQW pulses, it is possible to tap different electrical properties of the medium. For example, the application of a higher frequency (e.g. $1 \mathrm{kHz}$ ) allows us to reach the initial state of the electrification, since the ion motion is frozen on the time-scale of this experiment, no screening is

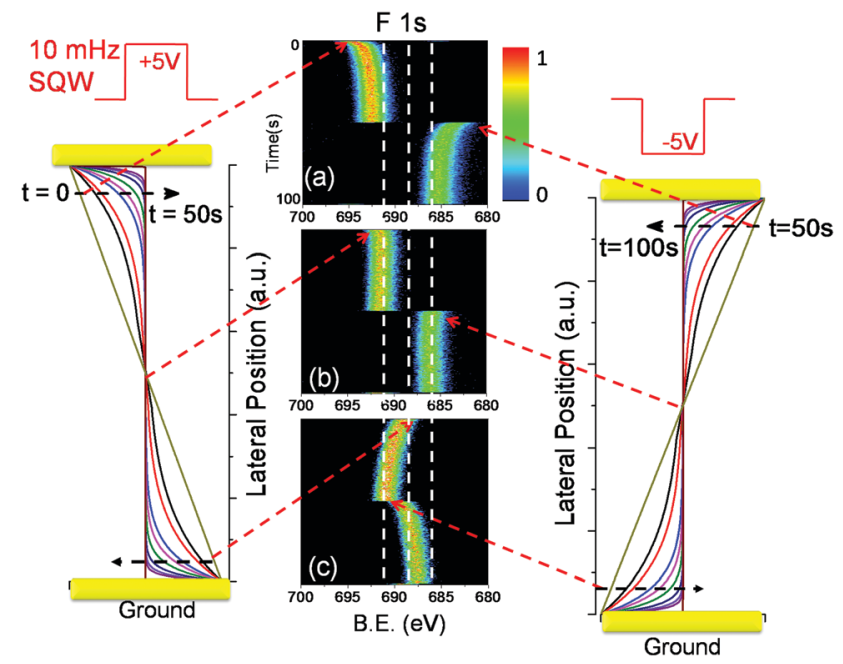

Fig. 4 Time-resolved XP spectra of the F1s region under $10 \mathrm{mHz} 5 \mathrm{~V}$ SQW excitation and recorded at three different lateral positions corresponding to points 1, 4 and 9 of Fig. 3. Schematic representation of the potential changes along the channel and at different times under positive and negative $5 \mathrm{~V}$ cycles. 


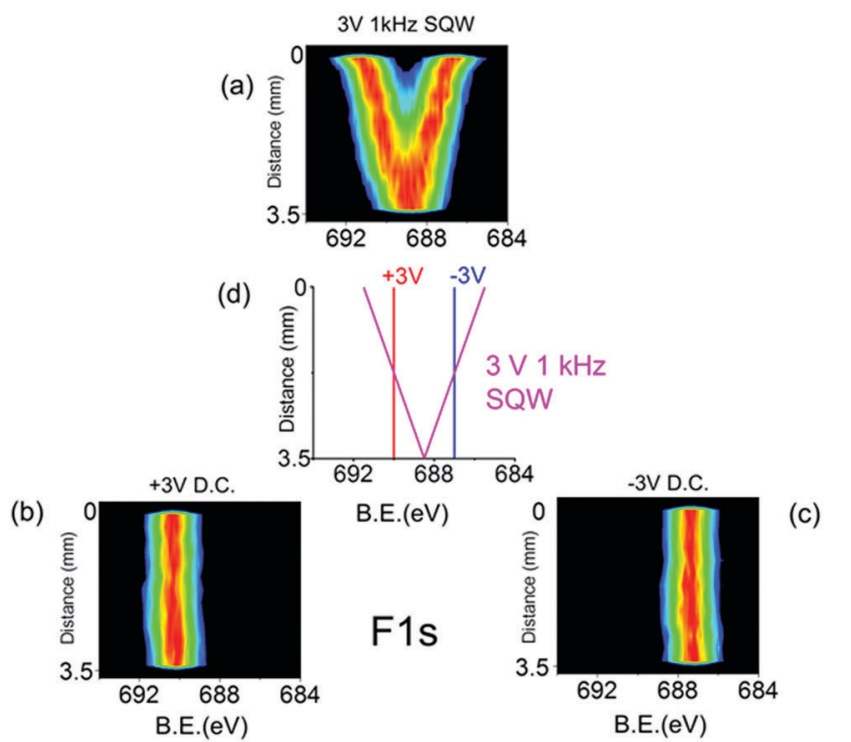

Fig. 5 (a) F1s region recorded in a line scan mode across the electrodes under $3 \vee 1 \mathrm{kHz}$ SQW excitation. (b and c) Similar data but recorded under +3 and $-3 \vee D C$ bias (the same spectra is also shown in Fig. 2). (d) A simple simulation of the F1s B.E. position under different stimuli.

effective and the applied voltage variations along the channel between the electrodes faithfully follow the simple resistive strip model ${ }^{53}$ as depicted in Fig. 5 (also see Fig. S4 in the ESI $\dagger$ ). Accordingly, the $1 \mathrm{kHz}$ SQW measurements correspond to zerotime onset (frozen), and the DC measurements correspond to the other extreme of the infinite time.

The observed voltage dependence, on the lateral position of the ionic liquid across the electrodes, can also be modeled with a simple network of resistors and two capacitors, as shown in Fig. 6. The capacitors at either side are modeling the electrochemical double layer on the metal-IL interface, and the resistor in the middle is modeling the IL infused polyethylene. To take into account the persistent $\sim 10 \mathrm{nA}$ current which was mentioned earlier, two resistors were also included, representing the unavoidable oxidation-reduction taking place on the symmetric gold electrodes, as the proposed leakage mechanism across the double layer capacitance. Yet another geometry where the large parallel resistor was placed across the two gold electrodes to represent a current leakage yields a very similar picture.

As can be seen in Fig. 6, switching the $+5 \mathrm{~V}$ pulse causes an upward jump, consequently an increase in the binding energy of the measured F1s, when monitored near the biased electrode (designated as position 1 in Fig. 3). Subsequent screening by the IL causes a decrease in time and brings it to the near equilibrium position of $+2.5 \mathrm{eV}$. In the negative cycle a reverse behavior is observed. When the corresponding behavior is monitored in a point near the grounded electrode (position 9 in Fig. 3), an opposite picture arises. Before switching the $+5 \mathrm{~V}$ cycle of the pulse, this point was at $-2.5 \mathrm{~V}$, the equilibrium potential under $-5 \mathrm{~V}$, and with the $+5 \mathrm{~V}$ pulse, and together with the screening action of the IL, its position starts moving towards positive directions, and descends to the near equilibrium position of $+2.5 \mathrm{~V}$. This simple simulation overlooks the fine details of charging at the interface and the ionic liquid surface but provides a basic understanding of the underlying polarization.

\section{Summary and conclusions}

Overall, several new experimental strategies using XPS have been presented to enable the extraction of new and important information, pertinent to the electrical properties of IL surfaces. ${ }^{62}$ First and foremost, the use of XPS to tap chemically addressed electrical information in a non-invasive fashion is pivotal, since it is directly obtained from the atomic constituents of the IL medium, rather than indirect information derivable from electrical and/or electrochemical methods. ${ }^{8,40}$ In this respect it can be compared with $\operatorname{IR}^{12,40}$ and $\operatorname{Raman}^{13}$ spectroscopic techniques. However, the quantitative nature of XPS brings an important advantage, similar to $\mathrm{NMR},{ }^{14,15}$ but again, with a broader applicability to all elements, except for hydrogen.
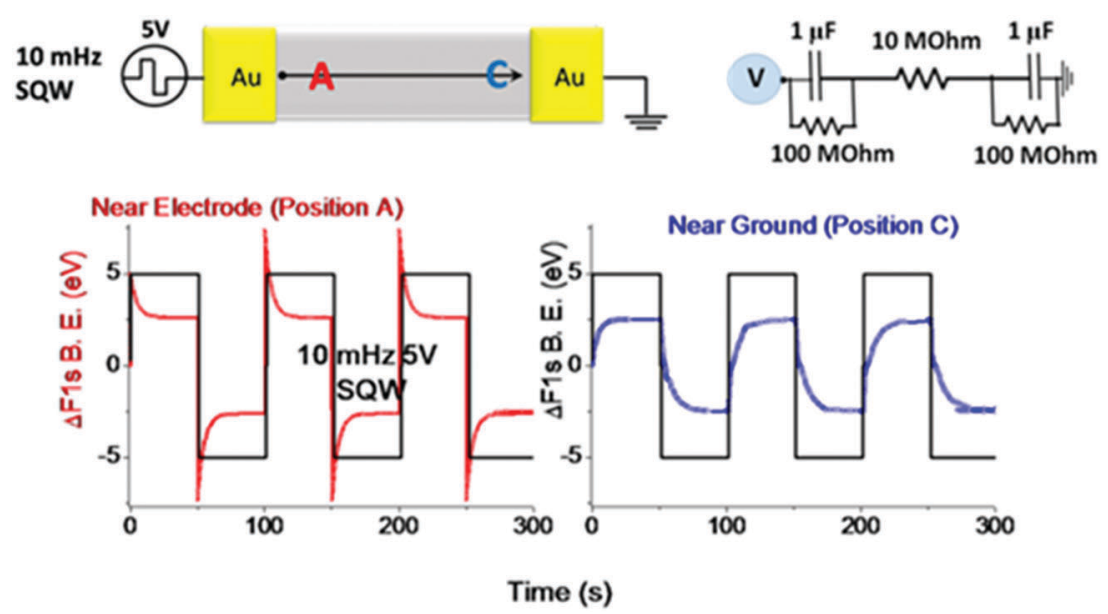

Fig. 6 Equivalent circuit model and simulation of the potential developed at two positions upon application of $5 \mathrm{~V} 10 \mathrm{mHz} \mathrm{SQW}$ pulses. The values of the resistor and the capacitors are chosen to match the experimental observations. 
Nevertheless, the slow response of the F1s signal (or other peaks) in tens-to-hundreds of seconds, as presented in Fig. 3, is in total agreement with recent experimental and theoretical work, ${ }^{8-34}$ and brings in complementary information related to ion dynamics. The data presented in Fig. 2 and simulated in Scheme 1, related to the formation of two similar but oppositely polarized EDLs at both electrodes, and the voltage drop at the metal electrode-IL medium interfaces, are new and direct experimental demonstration of the earlier theoretical prediction. ${ }^{60,61}$ Moreover, data presented in Fig. 3 and 4 on the laterally resolved information, retrieved from the chemically addressed XPS F1s signal, also bring a new and equally important finding, where it is shown that the device is partitioned into cathodically and anodically polarized regions and they meet at a neutral point in the middle. However, the most significant finding of our work is related to the fact that the effects of the electrode potential screening by the mobile ionic components are translated to an unexpectedly long lateral range of millimeters, reminiscent of soliton waves. ${ }^{63}$ Although our simple equivalent circuit model predicts such behavior, further theoretical and simulation studies are definitely needed.

In conclusion, this work describes several new routes, both in device formulation, and also in the use of the old XPS analysis technique with new tricks, to enable furthering our understanding about electrochemical concepts, including the very long range time and position effects of the electrical double layer formation, in a chemically addressed fashion.

\section{Acknowledgements}

This work was partially supported by the Scientific and Technological Research Council of Turkey (TUBITAK) Grant No. 212M051. CK also acknowledges the support from the European Research Council (ERC) Consolidator Grant ERC No. 682723 SmartGraphene. SS greatly acknowledges fruitful discussions with Dr Karl Doblhofer and Prof. Dr Ha.-Jo. Freund of the Fritz-Haber Institute of Berlin, Germany.

\section{References}

1 R. D. Rogers and K. R. Seddon, Science, 2003, 302, 792.

2 S. Zhang, N. Sun, X. He, X. Lu and X. Zhang, J. Phys. Chem. Ref. Data, 2006, 35, 1475.

3 P. Simon and Y. Gogotsi, Nat. Mater., 2008, 7, 845.

4 Y. Lu, K. Korf, Y. Kambe, Z. Tu and L. A. Archer, Angew. Chem., Int. Ed., 2014, 126, 498.

5 I. Osada, H. de Vries, B. Scrosati and S. Passerini, Angew. Chem., Int. Ed., 2016, 55, 500.

6 C. B. Byers, D. P. Nadappuram, K. Perry, K. McKelvey, A. W. Colburn and P. R. Unwin, Anal. Chem., 2015, 87, 10450.

7 W. I. S. Galpothdeniya, B. P. Regmi, K. S. McCarter, N. de Rooy, S. L. Siraj and I. M. Warner, Anal. Chem., 2015, 87, 4464.

8 C. Y. Peñalber and S. Baldelli, J. Phys. Chem. Lett., 2012, 3, 844.

9 S. Xu, S. Xing, S. S. Pei, V. Ivaništšev, R. Lynden-Bell and S. Baldelli, J. Phys. Chem. C, 2015, 119, 26009.
10 S. Baldelli, Acc. Chem. Res., 2008, 41, 421.

11 S. Baldelli, J. Phys. Chem. Lett., 2013, 4, 244.

12 K. Motobayashi, K. Minami, N. Nishi, T. Sakka and M. Osawa, J. Phys. Chem. Lett., 2013, 4, 3110.

13 K. Iwata, H. Okajima, S. Saha and H. O. Hamaguchi, Acc. Chem. Res., 2007, 4, 1174.

14 Z. X. Luo, Y.-Z. Xing, Y.-C. Ling, A. Kleinhammes and Y. Wu, Nat. Commun., 2015, 6, 1.

15 J. M. Griffin, A. C. Forse, W. Y. Tsai, P. L. Taberna, P. Simon and C. P. Grey, Nat. Mater., 2015, 14, 812.

16 E. Sloutskin, B. M. Ocko, L. Tamam, I. Kuzmenko, T. Gog and M. Deutsch, J. Am. Chem. Soc., 2005, 127, 7796.

17 M. Mezger, H. Schröder, H. Reichert, S. Schramm, J. S. Okasinski, S. Schöder, V. Honkimäki, M. Deutsch, B. M. Ocko and J. Ralston, Surf. Sci., 2008, 322, 424.

18 M. Mezger, B. M. Ocko, H. Reichert and M. Deutsch, Proc. Natl. Acad. Sci. U. S. A., 2013, 110, 3733.

19 A. Uysal, H. Zhou, G. Feng, S. S. Lee, S. Li, P. Fenter, P. T. Cummings, P. F. Fulvio, S. Dai and J. K. McDonough, J. Phys. Chem. C, 2013, 118, 569.

20 A. Uysal, H. Zhou, G. Feng, S. S. Lee, S. Li, P. T. Cummings, P. F. Fulvio, S. Dai, J. K. McDonough and Y. Gogotsi, J. Phys.: Condens. Matter, 2015, 27, 032101.

21 R. Hayes, N. Borisenko, M. K. Tam, P. C. Howlett, F. Endres and R. Atkin, J. Phys. Chem. C, 2011, 115, 6855.

22 S. A. Kislenko, I. S. Samoylov and R. Amirov, Phys. Chem. Chem. Phys., 2009, 11, 5584.

23 B. Bhargava and S. Balasubramanian, J. Am. Chem. Soc., 2006, 128, 10073.

24 R. M. Lynden-Bell, M. G. Del Popolo, T. G. Youngs, J. Kohanoff, C. G. Hanke, J. B. Harper and C. C. Pinilla, Acc. Chem. Res., 2007, 40, 1138.

25 Y. Wang, J. Phys. Chem. B, 2009, 113, 11058.

26 Y. Z. Su, Y. C. Fu, J. W. Yan, Z. B. Chen and B. W. Mao, Angew. Chem., Int. Ed., 2009, 121, 5250.

27 V. Ivaništšev, K. Kirchner, T. Kirchner and M. V. Fedorov, J. Phys.: Condens. Matter, 2015, 27, 102101.

28 M. V. Fedorov and A. A. Kornyshev, Chem. Rev., 2014, 114, 2978.

29 Y.-X. Zhong, J.-W. Yan, M.-G. Li, X. Zhang, D.-W. He and B.-W. Mao, J. Am. Chem. Soc., 2014, 136, 14682.

30 R. Atkin, N. Borisenko, M. Drüschler, F. Endres, R. Hayes, B. Huber and B. Roling, J. Mol. Liq., 2014, 192, 44.

31 A. Elbourne, S. McDonald, K. Voichovsky, F. Endres, G. G. Warr and R. Atkin, ACS Nano, 2015, 9, 7608.

32 N. Hjalmarsson, D. Wallander, S. Glavatskih, R. Atkin, T. Aastrup and M. W. Rutland, Nanoscale, 2015, 7, 16039.

33 D. T. Limmer, Phys. Rev. Lett., 2015, 115, 256102.

34 B. Rotenberg and M. Salanne, J. Phys. Chem. Lett., 2015, 6, 4978.

35 R. Hayes, G. G. Warr and R. Atkin, Chem. Rev., 2015, 115, 6357. 36 M. A. Gebbie, H. A. Dobbs, M. Valtiner and J. N. Israelachvilli, Proc. Natl. Acad. Sci. U. S. A., 2015, 112, 7432.

37 Y. Liu, C. Lu, S. Twigg, M. Ghaffari, J. Lin, N. Winograd and Q. M. Zhang, Sci. Rep., 2013, 3, 973.

38 Y. Yasui, Y. Kitazumi, R. Ishimatsu, N. Nishi and T. Kakiuchi, J. Phys. Chem. B, 2009, 113, 3273. 
39 Y. Yasui, Y. Kitazumi, R. Ishimatsu, N. Nishi and T. Kakiuchi, J. Phys. Chem. B, 2010, 114, 11141.

40 W. Zhou, S. Inoue, T. Iwahashi, K. Kanai, K. Seki, T. Miyamae, D. Kim, Y. Katayama and Y. Ouchi, Electrochem. Commun., 2010, 12, 672.

41 N. Nishi, Y. Hirano, T. Motokawa and T. Kakiuchi, Phys. Chem. Chem. Phys., 2013, 15, 11615.

42 E. F. Smith, I. J. Villar-Garcia, D. Briggs and P. Licence, Chem. Commun., 2005, 5633.

43 E. F. Smith, F. J. Rutten, I. J. Villar-Garcia, D. Briggs and P. Licence, Langmuir, 2006, 22, 9386.

44 A. W. Taylor, F. Qiu, I. J. Villar-Garcia and P. Licence, Chem. Commun., 2009, 5817.

45 K. R. Lovelock, I. J. Villar-Garcia, F. Maier, H. P. Steinrück and P. Licence, Chem. Rev., 2010, 110, 5158.

46 F. Qiu, A. W. Taylor, S. Men, I. J. Villar-Garcia and P. Licence, Phys. Chem. Chem. Phys., 2010, 12, 1982.

47 I. J. Villar-Garcia, E. F. Smith, A. W. Taylor, F. Qui, K. R. J. Lovelock and P. Licence, Phys. Chem. Chem. Phys., 2011, 13, 2797.

48 P. Licence, Angew. Chem., Int. Ed., 2012, 51, 4789.

49 C. Kolbeck, I. Niedermaier, A. Deyko, K. R. J. Lovelock, N. Taccardi, W. Wei, P. Wasserscheid, F. Maier and H. P. Steinrück, Chem. - Eur. J., 2014, 20, 3954.
50 S. Men, D. S. Mitchell, K. R. Lovelock and P. Licence, ChemPhysChem, 2015, 16, 2211.

51 T. Matsuda, N. Taccardi, J. Schwegler, P. Wasserscheid, H. P. Steinrück and F. Maier, ChemPhysChem, 2015, 16, 1873.

52 H. Sezen and S. Suzer, Thin Solid Films, 2013, 534, 1.

53 C. Kocabas and S. Suzer, Anal. Chem., 2013, 85, 4172.

54 B. Bozzini, M. Amati, L. Gregoratti, M. K. Abyaneh, M. Prasciolu, A. L. Trygub and M. Kiskinova, J. Phys. Chem. C, 2012, 116, 23188.

55 A. Foelske-Schmitz, D. Weingarth, H. Kaiser and R. Kötz, Electrochem. Commun., 2010, 12, 1453.

56 A. Foelske-Schmitz, P. W. Ruch and R. Kötz, J. Electron Spectrosc. Relat. Phenom., 2010, 182, 57.

57 D. Weingarth, A. Foelske-Schmitz, A. Wokaun and R. Kötz, Electrochem. Commun., 2011, 13, 619.

58 M. F. El-Kady and R. B. Kaner, Nat. Commun., 2013, 4, 1475.

59 Z.-S. Wu, K. Parvez, X. Feng and K. Mullen, Nat. Commun., 2013, 4, 2487.

60 C. Merlet, M. Salanne, B. Rotenberg and P. A. Madden, J. Phys. Chem. C, 2012, 115, 16613.

61 T. Fujimoto and K. Awaga, Phys. Chem. Chem. Phys., 2013, 15, 8983.

62 K. R. Lovelock, Phys. Chem. Chem. Phys., 2012, 14, 5071.

63 https://en.wikipedia.org/wiki/Soliton. 